\title{
Lateral Approach Technique to Minimize Bladder Injury During Abdominal Hysterectomy in Cases with Previous Cesarean Sections: An Observational Study
}

\author{
Ibrahim Saif Elnasr* \\ Obstetrics and Gynecology Department, Menoufia University-Menoufia governorate, Egypt
}

Submission: October 04, 2018 ; Published: October 25, 2018

*Corresponding author: Ibrahim Saif Elnasr, Obstetrics and Gynecology Department, Faculty of Medicine, Menoufia University-Menoufia governorate, Egypt, Tel: +2 01003086747; Email: Ibrahim_saif@yahoo.com

Abstract

Objective: to evaluate the safety and efficacy of Lateral approach technique to avoid bladder injury during total abdominal hysterectomy in cases with previous Cesarean section.

Methods: This prospective observational study included 150 patients with previous cesarean deliveries who were candidates for total abdominal hysterectomy due to various indications. Enrolled patients were divided in two groups; Group 1 included 75 patients to whome the urinary bladder was dissected from the uterus by classical central approach and Group 2 included 75 patients to whome the bladder was dissected from the uterus by lateral approach technique. The rate of intraoperative bladder injury, operative time and late urological complications were the main outcome measures. Data was collected and tabulated.

Results: there was significant statistical difference between two groups as regarding urinary bladder injury $(<0.05)$ with only one case of bladder injury in group 2 compared to 10 cases in group one. The duration of the procedure was shorter in group 2 ( $<<0.001$ ).Group one Patients need excessive analgesia $(<0.05)$, post-operative fever increased in group one $(<0.001)$. Group one Patients need blood transfusion $(<0.05)$ and hospital stay more than group $2(<0.001)$.

Conclusion: lateral approach technique for bladder dissection was safer and faster in preventing bladder injury during total abdominal hysterectomy compared to the classical central approach. Larger multicenter trials are warranted to enforce or refute these findings.

Keywords: Lateral approach; Bladder injury; Total abdominal hysterectomy; Previous Cesarean section

\section{Introduction}

Hysterectomy is one of the most common operations performed in the United States. Approximately 600,000 per year, It was reported that uterine fibroid considered the Most common indications for hysterectomy ( $40.7 \%$ ) followed by endometriosis $(17.7 \%)$ and prolapse (14.5\%) [1].

Multiple surgical approaches may be applied to perform Hysterectomy including abdominal approach, vaginal approach, laparoscopic assisted vaginal approach, laparoscopic and robotic assisted approaches [2]. However, analysis of U.S. surgical data shows that abdominal hysterectomy is the most common rout for hysterectomy and performed in $66 \%$ of cases, vaginal hysterectomy in $22 \%$ of cases, and laparoscopic hysterectomy in $12 \%$ of cases [3].

Many complications may occur during Hysterectomy. Urological injuries considered common complication during hysterectomy especially in patients with history of previous caesarian section [4]. As reported by Sheth, et al. [5], Bladder injury considered the commonest urological complication during hysterectomy and the increasing number of cesarean deliveries nowadays may contribute to a higher incidence of bladder injuries in subsequent hysterectomies due to dense vesicocervical adhesions and distorted anatomy [5]. For women with histories of cesarean delivery, the odds ratios for bladder injury were 7.50 for laparoscopic-assisted vaginal hysterectomy (LAVH), 1.26 for total abdominal hysterectomy (TAH), and 3.00 for transvaginal hysterectomy [6].

Adhesions between the bladder and the lower uterine segment after cesarean section commonly limited to vesicocervical space with sparing of parametrial space. W-c Chang, et al. [7] considered dissecting the bladder from lateral to medial through the broad ligament decrease risk of bladder injury during laparoscopic assisted vaginal hysterectomy (LAVH) [8]. This study was conducted to evaluate the efficacy and safety of Lateral approach technique through the broad ligament to avoid bladder injury during total abdominal hysterectomy in cases with previous Cesarean section. 


\section{Materials and Methods}

After the study protocol was reviewed and approved by the local ethics committee at Menoufia University hospital, this prospective observational study was performed at the Obstetrics and Gynecology department at Menoufia university hospital, Shibin El-kom city, Menoufia governorate, Egypt in the period between January 2017 till August 2018.

All procedures performed in the current study involving human participants were in accordance with the ethical standards of the Menoufia Fcaulty of Medicine research committee and with the 1964 Helsinki declaration and its later amendments. The study was approved by Institutional review board of Obstetrics and Gynaecology department, Letter Number (326H/2016).

The cases of urologic injuries during total abdominal hysterectomy were identified from the operating room record book, in patient room record book and departmental monthly audit reports. Demographic and clinical data collected included age, parity, body mass index, Number of cesarean deliveries and indications for hysterectomy Operative data collected included Type of anesthesia, Operative time, Ureter and Small intestine injury, Blood transfusion, Bladder injury and blood loss. Postoperative data collected included venous thromboembolism, surgical site infection, reoperation, Request for additional analgesia, Fever and Hospital stay.

In our study we excluded cases underwent total abdominal hysterectomy without previous CS scar, vaginal hysterectomy, laparoscopic assisted vaginal hysterectomy, laparoscopic hysterectomy and cesarean hysterectomy, 150 women with previous cesarean deliveries who were candidates for total abdominal hysterectomy enrolled and was allocated into two groups

Group 1: include 75 patients while the bladder was dissected from the uterus by classical central approach

Group 2: include 75 patients while the bladder was dissected from the uterus by lateral approach technique

\section{The Following Surgical Steps was Performed for all Patients}

The patients were in the dorsal position. Sterilization, catheterization and toweling were done. After general or regional anesthesia skin incision was conducted vertically or horizontally according to the indication for hysterectomy. The incision was made to cut through skin and muscle of the abdomen. The general condition of the abdomen was inspected. Both round ligament and infundiplopelvic ligament was clamped and ligated in both side. The space between anterior and posterior leaflet of the broad ligament was opened by surgical scissor then easily through blunt dissection by finger. The key was that we stayed as lateral as possible and not moved too medially toward the uterus to avoid injury to uterine vessels with care to vessels in the lateral pelvic wall. The posterior leaflet of the broad ligament was cut toward the origin of uterosacral ligament after carful dissection to avoid ureteric injury.

Once adequate area of posterior leaflet was dissected and cut and adequate window was created in the broad ligament, the anterior leaflet of the broad ligament was elevated by artery forceps then we swept the index finger of the right hand from lateral to medial to define the midline adhesions secondary to previous cesarean deliveries. During creation of lateral window the uterus was taken upward and to the opposite side then it was centralized upward posteriorly to give more counter traction. After clearly identifying bladder wall we pushed down the bladder away before cutting any adhesion band under direct vision and finger guidance. We preferred to push bladder entirely from one side (started with Rt side) so once adequate amount of dissection was conducted we moved easily to the opposite side to complete bladder dissection. Both uterine arteries were clamped and ligated then total hysterectomy was performed as usual manner.

Foleys catheter was inserted to all patients which removed after patients were fully mobilized. All patients received routine intraoperative and post-operative care for abdominal hysterectomy and discharged from the hospital after catheter removal and returning of intestinal motility. All the patients were monitored at the outpatient clinic 1 week after operation to evaluate the wound for surgical site infection and any urological complaint. Then after one month to evaluate for possibilities of late urological complication as genitourinary fistula.

\section{Outcome measures}

\section{The primary outcome measures}

Efficacy and safety of the procedure (defined as proper bladder dissection from the uterus without bladder injury).

\section{The secondary outcome measures}

Duration of the procedure, operative time, blood loss and late urological complications as genitourinary fistula and ureteric injury.

\section{Statistical analysis}

The data collected were tabulated \& analyzed by SPSS (statistical package for the social science software) statistical package version 22 on personal compatible computer. Quantitative data was analyzed by applying student t- test or Mann-Whitney test as required while qualitative data was analyzed by applying Chi-square test and Fisher's exact test as required with a significance level of $P$ value less than 0.05 .

\section{Results}

During the study period out of 170 hysterectomies were conducted in our unites, 20 cases vaginal hysterectomy, 75 cases abdominal hysterectomy in patient with no previous scar and 75 cases abdominal hysterectomy in patients with previous scar. Table 1 represents patients' characteristics. There was no 


\section{Journal of Gynecology and Women's Health}

significant difference between both group regarding age, parity, body mass index, Number of cesarean deliveries and indication for hysterectomy $(\mathrm{p}>0.05)$.

Table1: Patient characteristics:

\begin{tabular}{|c|c|c|c|c|}
\hline & Group $1(n=75)$ & Group $2(n=75)$ & Student t-test & P-value \\
\hline Age (years) & $45.6 \pm 20.1$ & $46.2 \pm 19.8$ & 0.18 & $>0.05$ \\
\hline Parity & $2.2 \pm 1.6$ & $2.3 \pm 1.4$ & $0.41^{*}$ & $>0.05$ \\
\hline BMI $\left(\mathrm{Kg} / \mathrm{m}^{2}\right)$ & $25.4 \pm 4.1$ & $25.7 \pm 3.9$ & 0.46 & $>0.05$ \\
\hline \multicolumn{5}{|c|}{ Number of cesarean deliveries } \\
\hline -One CS & 15 & 17 & & \multirow{7}{*}{$>0.05$} \\
\hline -Two CS & 30 & 25 & & \\
\hline -Three CS & 12 & 13 & & \\
\hline -Four CS & 10 & 10 & & \\
\hline -Five CS & 4 & 3 & & \\
\hline -Six CS & 3 & 5 & & \\
\hline -Seven CS & 1 & 2 & & \\
\hline \multicolumn{5}{|c|}{ Benign lesions } \\
\hline - Uterine fibroid & 30 & 25 & & \multirow{7}{*}{$>0.05$} \\
\hline - Dysfunctional uterine & 15 & 14 & & \\
\hline bleeding - Adenomyosis & 6 & 8 & & \\
\hline - Chronic pelvic pain & 0 & 1 & & \\
\hline - Tubo-ovarian abscess & 1 & 3 & & \\
\hline - Benign ovarian mass & 5 & 4 & & \\
\hline -Endometrial hyperplasia & 7 & 9 & & \\
\hline \multicolumn{5}{|c|}{ Malignant lesions } \\
\hline - ovarian mass & 5 & 4 & & \multirow{4}{*}{$>0.05$} \\
\hline -Atypical endometrial hyperplasia & 2 & 3 & & \\
\hline -Endometrial carcinoma & 3 & 4 & & \\
\hline Cervical carcinoma & 1 & 0 & & \\
\hline
\end{tabular}

*Mann Whitney test, CS=Cesarean section

Table 2 revealed the operative data. There was no significant difference between both group regarding age Type of anesthesia Operative time, Ureter and Small intestine injury ( $p>0.05)$. There was significant difference between both group regarding Blood transfusion $(<0.05)$. there was significant difference

between both group as regarding Bladder injury with marked improvement in group 2 one case of bladder injury in contrast to 10 cases in group $2(<0.05)$. There was highly significant difference between both group as regarding blood loss $(<0.001)$.

Table 2: Patient characteristics:

\begin{tabular}{|c|c|c|c|c|c|}
\hline \multicolumn{7}{|c|}{} & Group 1 (n=75) & Group 2 (n=75) & Chi square test & P-value & Odd's ratio at 95\% CI \\
\hline \multicolumn{7}{|c|}{ Type of anesthesia } \\
\hline -General & 25 & 20 & 0.51 & $>0.05$ & $1.38(0.68-2.77)$ \\
\hline -Regional & 50 & 55 & & \\
\hline Operative time (minutes) & $160.9 \pm 58.8$ & $162.1 \pm 58.6$ & $0.81 *$ & $>0.05$ & \\
\hline Estimated blood loss (mL) & $880.8 \pm 480.6$ & $870.2 \pm 490.4$ & $5.18^{*}$ & $<0.001$ & \\
\hline \multicolumn{7}{|c|}{ Organ injury } & 6.28 & $<0.05$ & $11.38(1.42-91.3)$ \\
\hline -Bladder injury & 10 & 1 & 0 & $>0.05$ & \\
\hline -Ureteric injury & 2 & 0 & 0 & $>0.05$ & $2.03(0.18-22.8)-$ \\
\hline -Small intestine injury & 1 & 0 & 2.89 & $<0.05$ & $2.32(0.97-5.5)$ \\
\hline Blood transfusion & 18 & 9 & & \\
\hline
\end{tabular}

Table 3 shows postoperative data. There was no significant SSI=Surgical site infection and reoperation $(>0.05)$. There was difference between both group VTE=Venous thromboembolism, significant difference between both group regarding Request 
for additional analgesia $(<0.05)$. There was highly significant difference between both group as regarding Fever and Hospital stay $(<0.001)$.

Table 3: Post-operative data.

\begin{tabular}{|c|c|c|c|c|c|}
\hline & Group 1 (n=75) & Group 2 (n=75) & Chi square test & P-value & Odd's ratio at 95\% CI \\
\hline Request for additional analgesia & 36 & 18 & 8.36 & $<0.05$ & $2.92(1.46-5.87)$ \\
\hline \multicolumn{7}{|c|}{ Postoperative complications } \\
\hline -Fever & 9 & 14 & 16.81 & $<0.001$ & $4.72(2.26-9.86)$ \\
\hline -VTE & 4 & 2 & $0.17^{*}$ & $>0.05$ & $2.06(0.37-11.6)$ \\
\hline -SSI & 6 & 4 & $0.11^{*}$ & $>0.05$ & $1.54(0.42-5.71)$ \\
\hline Re-operation & 4 & 1 & $0.8^{*}$ & $>0.05$ & $4.11(0.45-37.7)$ \\
\hline Hospital stay (days) & $8.6 \pm 5.1$ & $4.8 \pm 2.1$ & $5.59 \dagger$ & $<0.001$ & \\
\hline
\end{tabular}

${ }^{*}$ Fischer's exact test, †Student t-test, VTE=Venous thromboembolism, SSI=Surgical site infection.

\section{Discussion}

Hysterectomy remains the most common gynecologic procedure in the United States and all over the world; although Minimally invasive approaches to hysterectomy have welldocumented advantages, yet abdominal hysterectomy remains the most common mode of access, accounting for more than $60 \%$ of all hysterectomies performed in the United States [6].

Multiple cesarean deliveries are associated with an increased risk of pelvic adhesive disease and difficulty with bladder dissection during subsequent gynecologic surgery especially hysterectomy. The bladder dome adhered to the lower uterine segment requires meticulous dissection to avoid injury. Cystostomy occurs in greater than $20 \%$ of women with more than 3 prior cesarean deliveries [7].

Urinary bladder injury considered important surgical hazardse specially during hysterectomy and cesarean section. With regard to bladder injury, the dome of the bladder is commonly involved in injury during total hysterectomy. Nearly two thirds of all bladder injuries occur during gynecological surgery and most of these complications occur during surgeries via abdominal route compared to vaginal route [8].

Urinary bladder injuries due to obstetric and gynecologic surgery are normally divided into two categories: acute complications such as bladder laceration that can be identified immediately during the operation, and chronic complications such as vesico-vaginal fistula which can occur later on. To avoid these injuries the gynecologist must have an accurate understanding of pelvic anatomy, use a meticulous and methodical surgical technique, and maintain a constant high degree of vigilance [9].

Most adhesions between the bladder and the loweruterine segment after cesarean delivery are located in the vesico-cervical space, which is in the middle of the operation field flanked by bilateral parametrial space inside the broad ligaments. The idea of trying to go from lateral to medial toward the bladder is that in almost cases of previous section the fibrous scar tissue related to uterus only and lateral space not affected so we the bladder was separated and fibrous tissue was cut under vision and finger guidance.
In our study symptomatic uterine fibroid was the most common indication for hysterectomy and other indications listed in Table 1. All patient passed flatus and had oral fluid intake at next day of surgery. We noticed that blood loss and blood transfusion was less in group 2 this may be due to reaching a good plane for bladder dissection which was less vascular without injury to any pelvic venous plexus.

There was significant difference between both group as regarding Bladder injury with marked improvement in group 2 where one case of bladder injury in contrast to 10 cases in group $2(<0.05)$ this is may be due to proper identification of bladder anatomy. This in consistence with the study conducted by WC Chang et al. [7] where 50 patients with vesicocervical adhesion after previous cesarean deliveries who underwent laparoscopic assisted vaginal hysterectomy After incorporation of the lateral intervention method into the LAVH procedures, no bladder injury occurred among any of the 50 patients.

Our results not consistence with the study conducted by Tarek R et al. [9] where 570 patients had abdominal hysterectomy The frequency of bladder injury was higher being $2.3 \%$. This high incidence may be due to bladder dissection in classic approach. All cases with bladder injury during total abdominal hysterectomy were diagnosed intraoperatively and immediately repaired by urological consultant in two layers with $2 / 0$ vicryl with Foleys catheter drainage for 10 days. Only one case (group 1) presented after one month from surgery complaining of urine leak, investigated and diagnosed as having vesicovaginal fistula which repaired after three months from the surgery. In this study we had some technical difficulties in the first few case but in rapid fashion the surgical team became familiar with the technique and short operative time for abdominal hysterectomy was achieved.

\section{Conclusion}

We founded that lateral approach technique of bladder dissection was very useful in preventing bladder injury in our cases and also was very easy to be performed. Larger multi center trials are warranted to enforce or refute these findings. We recommend proper training of this approach by all staff and application of this approach to other cases as cesarean hysterectomy in cases of placenta accreta. 


\section{Acknowledgement}

The author would like to acknowledge the contribution of residents and nursing staff at the operating room and Urology staff of Menoufia University hospital.

\section{References}

1. Whiteman MK, Hillis SD, Jamieson DJ, Morrow B, Podgornik MN, et al (2008) In patient hysterectomy surveillance in the United States, 20002004. Am J Obstet Gynecol 198(1): 34e-1-7.

2. Nieboer TE, Johnson N, Lethaby A, Tavender E, Curr E, et al. (2009) Surgical approach to hysterectomy for benign gynecological disease. Cochrane Database Syst Rev 8(3): CD003677.

3. Wu JM, Wechter ME, Geller EJ, Nguyen TV, Visco AG, et al. (2007) Hysterectomy rates in the United States, 2003. Obstet Gynecol 110(5): 1091-1095.

4. Cosson M, Lambaudie E, Boukerrou M, Querleu D, CG Vaginal, et al. (2001) laparoscopic, or abdominal hysterectomies for benign disorders: immediate and early postoperative complications. Eur J Obstet Gynecol Reprod Biol 98(2): 231-236.

5. Sheth SS, Malpani A (1995) Vaginal hysterectomy following previous cesarean section. Int J Gynecol Obstet 50(2): 165-169.

6. Rooney CM, Crawford AT, Vassallo BJ, Kleeman SD, Karram MM, et al. (2005) Is previous cesarean section a risk for incidental cystotomy at the time of hysterectomy? Acase-controlled study. Am J Obstet Gynecol 193(6): 2041-2044.

7. W-Cchang, Hsu, Sheu BC, Huang SC, Torng PL, et al. (2008) Minimizing bladder injury in laparoscopically assisted vaginalhy sterectomy among women with previous cesarean sections. Surg Endosc 22: 171176.

8. Aronson MP, Bose TM (2002) Urinary tract injury in pelvic surgery. Clin Obstet Gynecol 45(2): 428-438.

9. Abbas TR, Osman MM (2011) Urological Injuries during Obstetric and Gynecological Surgical Procedures: Two Centers Experience. Med J Cairo Univ 79(1): 261-265. 